\title{
Nozzle Design of Hot Strip Steel Temperature Measurement Device and Analysis of its Flow Field Characteristics
}

\author{
Shui Xuan Chen, Gang Lu and Jian Min $\mathrm{Xu}^{*}$
}

\author{
School of Mechanical and Automotive Engineering, Xiamen University of Technology, Xiamen 361024, China
}

\begin{abstract}
A kind of new hot strip steel temperature measuring device has been designed. The mathematical model and the geometric model of the nozzle flow field have been established based on computational fluid dynamics theory. The impact of equalizing groove and nozzle structure on the nozzle system characteristics is researched in detail using fluid dynamics software FLUENT. The research results show that: the nozzle pressure groove can improve the gas flow properties of the nozzle and the nozzle can meet the design requirements. The nozzle gap, the inlet pressure of nozzle, the lower edge diameter of the nozzle, nozzle diameter and chamfer structure have different effects on flow field characteristics and nozzle flow. The study provides a theoretical basis for designing hot strip steel temperature measurement devices and the nozzle of purge.
\end{abstract}

Keywords: Hot strip temperature measuring device, computational fluid dynamics, nozzle, flow field characteristics.

\section{INTRODUCTION}

In the process of producing and controlling the hot-rolled strip steel, the strip steel temperature is one of the important factors that directly affect the accuracy of the final product size and the organizational performance. In the strip rolling process, prediction and control of temperature are key factors that affect the strip rolling speed, rolling force and the roll gap. According to the design of hot-rolled strip steel temperature measurement devices, high-pressure gas is ejected from the nozzle in the process of measuring temperature. The anhydrous bare area is produced when the cooling water on the strip steel surface is blown away. As can be seen from the measurement principle of the temperature measuring device, the nozzle plays a very crucial role in this temperature measuring program and its performance directly affects the performance of temperature measuring device. Therefore it is necessary to carry out the research of nozzle flow field in order to obtain some theoretical basis for designing the nozzle. There are many scholars that have conducted a detailed study on the nozzle flow characteristics. TAN Bao Hui, FENG Zhi Hua et al. [1] conducted the numerical simulation of the auxiliary nozzle flow field and obtained the velocity and pressure distribution profile curve of the auxiliary nozzle. Ruanhong Yan et al. [2] designed a combination of supersonic nozzle and analyzed the nozzle flow field. Hu Jun et al. [3] analyzed the relationship between the auxiliary flow field structure of supersonic gas jet nozzle and nozzle throat corner. They simplified the structure of the supersonic nozzle. Long Xinping et al. [4] conducted the numerical simulation and analysis of jet pump flow field and studied the vortex flow distribution of the internal pipe. Xing Shikai, et al. [5]

*Address correspondence to this author at the School of Mechanical and automotive Engineering, Xiamen University of Technology, Xiamen, 361024, P.R. China; Tel: 13459263068; E-mail: xujianmin1020@163.com conducted the numerical simulation of nozzle angle characteristics using three-dimensional numerical simulation technology. Jubao Liu et al. [6] conducted numerical simulation of abrasive water jet nozzle using CFD software CFX. Wang Guan Chao et al. [7] conducted flow field numerical simulation of air jet loom main nozzle using fluid dynamics software NUMECA. Wang Xu et al. [8] designed a special nozzle for cleanup robot and analyzed the flow field inside the nozzle to verify the reasonableness of nozzle design using software FLUENT. Guo jian et al. [9] studied the flow field of the ordinary ejector and a new ejector using numerical simulation method. Chen Qinghua and Zhang Guoshu [10] simulated the three-dimensional flow field inside the spray nozzle using software FLUENT. Zhang Zhen and Zhang Qiao Fang [11] conducted the flow field simulation for the high-pressure nozzle of submerged and non-submerged jet. Maokai Tian et al. [12] conducted the flow field simulation inside the injector using analysis software ANSYS (FLOTRAN).

In this paper, the impact of the nozzle gap, the inlet pressure of nozzle, the lower edge diameter of the nozzle, the nozzle diameter and the chamfer structure on the flow field characteristics and nozzle flow are researched in detail.

\section{DESIGN OF A NEW HOT STRIP STEEL TEMPERATURE MEASUREMENT DEVICE}

The new hot strip steel temperature measuring device is shown in Fig. (1). The temperature measuring device can be moved to any position of hot-rolled layer cold zone. The temperature measuring device can measure the surface temperature of the steel strip without any contact and any collision with the steel strip. The temperature measuring device includes the trolley (2) with bottom mounted rollers (1). On one hand, it is used to move the entire temperature measurement device, and on the other hand, it is used to support and fix the entire temperature measurement device when measuring temperature. The trolley is moved to one 
side of the cold zone baffle layer (7). The horizontal position of the platform is ensured by adjusting the mounting platform (3) on the trolley. The supporting arm (6) connects to the mounting platform by a rotation shaft. The support arm can be rotated to a horizontal position or any other position by the cylinder (4). Purge (12) is connected to the support arm by the parallelogram mechanism (11). The agency can ensure that the purge is not swinging and the purge is always perpendicular to the strip surface. The purge position can be controlled by the positioning motor installed inside the transmission case (8). The purge contains the nozzle (13) with a special aerodynamic shape, the bottom surface of which is parallel to the steel strip surface. According to aerodynamics, aerodynamic nozzle maintains a certain distance from the steel strip surface due to the force balance.

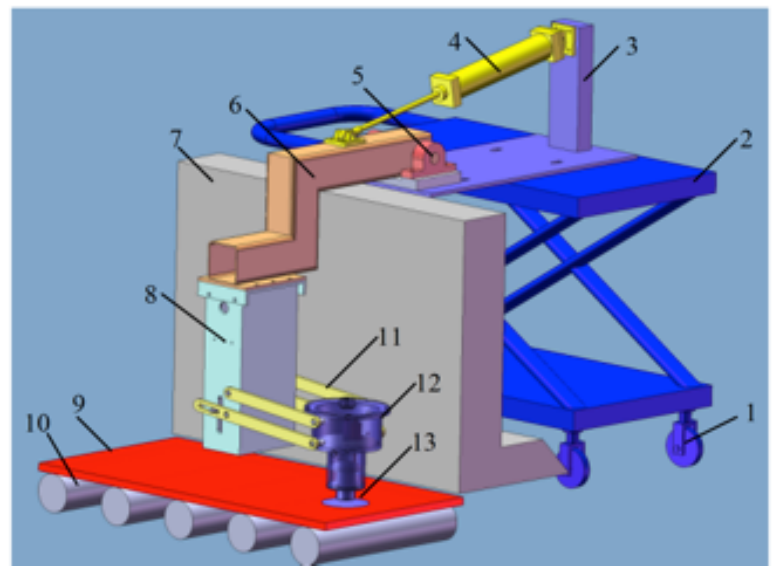

Fig. (1). Portable non-contact temperature measuring device.

Position control of purge and design of pneumatic nozzle are the core technologies of the temperature measuring device. Pneumatic nozzle is the core element of purge. A dry surface of the steel strip is formed when the compressed air from the nozzle blows into the aqueous layer on the steel strip. A pneumatic air cushion effect is formed between the nozzle and the sports strip by gas flow.

\section{THE STRUCTURAL DESIGN OF PURGE}

Fig. (2) shows the structure diagram and a cross-sectional view of the purge. Temperature measuring device mainly consists of nozzle mechanism, purge cavity, infrared temperature sensor, displacement sensor and mechanical device attached. In the process of measuring temperature, high-pressure gas from the nozzle is sprayed on the steel strip. Cushion effect formed because of high-pressure gas can levitate the nozzle. The cooling water high-pressure air is blown on the strip surface to form a dry area. A dry area is formed when high-pressure air blows out of the cooling water and onto the steel strip surface. Strip temperature of dry area can be obtained by infrared temperature sensor in order to achieve accurate surface temperature measurement of the steel strip.

\section{FLOW FIELD ANALYSIS OF NOZZLE}

\subsection{Mathematical Model}

Gas flow velocity inside the nozzle reaches $100 \mathrm{~m} / \mathrm{s}$ or more. Due to the high Reynolds number, the air movement inside the nozzle is turbulent. (a) Schematic structure

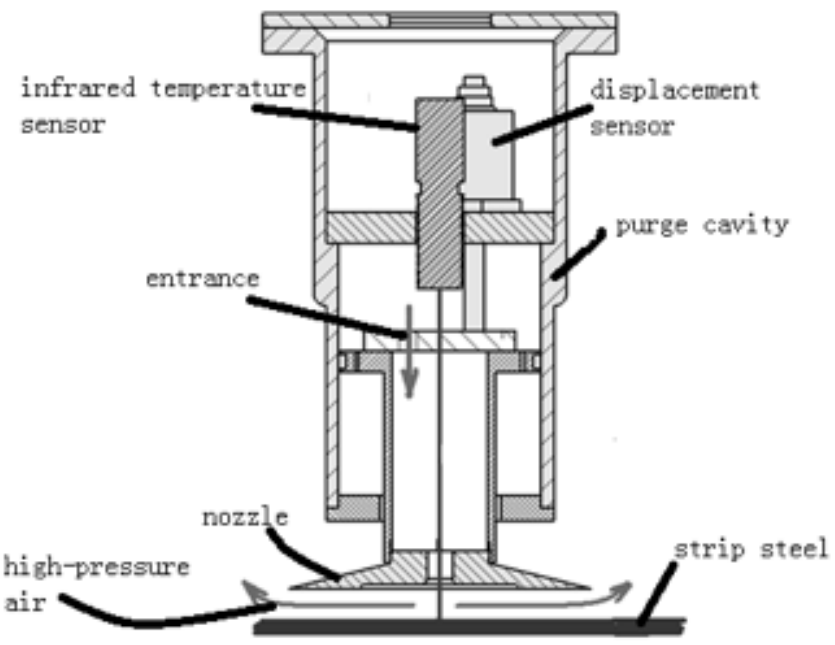

(b) A cross-sectional view of three-dimension

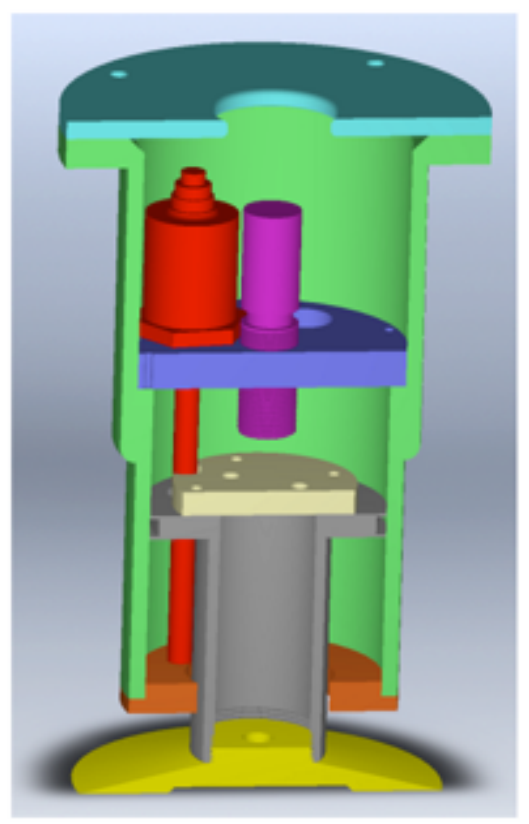

Fig. (2). Purge

\subsubsection{The Basic Equations}

In the Cartesian coordinate system, compressible Newtonian equations of fluid motion are expressed as follows:

\subsubsection{Mass Conservation Equations (Continuity} Equation)

$\frac{\partial \rho}{\partial t}+\frac{\partial \rho u_{j}}{\partial x_{j}}=0$

\subsubsection{The Momentum Conservation Equation}

$\frac{\partial \rho u_{i}}{\partial t}+\frac{\partial \rho u_{i} u_{j}}{\partial x_{j}}=-\frac{\partial p}{\partial x_{i}}+\frac{\partial \tau_{i j}}{\partial x_{j}}+\rho f_{i}$

The Navier-Stokes $(\mathrm{N}-\mathrm{S})$ equation is mentioned above. Where $u_{j}$ is the gas velocity in the direction of the 
component $x_{j}, \rho$ is the density of the gas, $p$ is the pressure, $\tau_{i j}$ is a Newtonian fluid viscous stress tensor, and $f$ is the quality of the force strength. Heat exchange is not considered here, so the energy equation can be omitted, therefore the constitutive equation is as follows.

$$
\tau_{i j}=\mu\left(\frac{\partial u_{i}}{\partial x_{j}}+\frac{\partial u_{j}}{\partial x_{i}}\right)-\frac{2}{3} \frac{\partial u_{k}}{\partial x_{k}} \delta_{i j}
$$

where $\mu$ is the dynamic viscosity coefficient of gas, it is physical constant, $\delta_{i j}$ is Kornecker delta function.

\subsubsection{Closed Equation}

The average equation is rewritten as:

$$
\frac{\partial}{\partial t}\left(\bar{\rho} \widetilde{u_{i}}\right)+\frac{\partial}{\partial x_{j}}\left(\bar{\rho} \widetilde{u_{i}} \widetilde{u_{j}}\right)=-\frac{\partial \bar{p}}{\partial x_{i}}+\frac{\partial \tau_{i j}^{e f f}}{\partial t}+\bar{\rho} \bar{f}
$$

where $\tau_{i j}^{e f f}=\overline{\tau_{i j}}+\tau_{i j}^{t}$ is the effective shear stress; $\overline{\tau_{i j}}$ is molecular viscous shear stress and $\tau_{i j}^{t}=-\rho \overline{\mu_{i}^{\prime \prime} \mu_{j}^{\prime \prime}}$ is called the turbulent shear stress or Reynolds stress. General functional form of Reynolds stress is as follows.

$$
\tau_{i j}^{t}(x, t)=\tau_{i j}\left[x, t ; \bar{U}, u^{\prime}(y, 0), u^{\prime \Sigma}\left(y^{\Sigma}, s\right)\right]
$$

where $\Sigma$ refers to the flow of geometric borders, $\bar{U}$ is the average velocity field. Eddy viscosity model (EVM) is commonly used in engineering turbulence model, its general form is as follows.

$$
\tau_{i j}^{t}=v_{t}\left(\frac{\partial \overline{u_{i}}}{\partial x_{j}}+\frac{\partial \overline{u_{j}}}{\partial x_{i}}\right)-\frac{2}{3} k \delta_{i j}
$$

where $v_{t}$ is the eddy viscosity coefficient (eddy viscosity), the amount to be modeled and turbulent kinetic energy. Here the enclosed control equations are completed by standard $k-\varepsilon$ model.

\subsection{Establishment of Geometric Model}

The cavity structure formed between the nozzle and the steel strip is shown in Fig. (3). Air flow acts on the steel strip through the nozzle chamber. The structural parameters of the nozzle are: nozzle length $\mathrm{L}$, the nozzle diameter $\mathrm{d} 1$, the diameter of the nozzle on the surface $d$, the diameter of the equalizing groove D1, the lower edge diameter of the nozzle, the gap between the nozzle and the strip $h$, pressure groove depth $\mathrm{h} 1$.

In the simulation, intake pressure $P=2 \times 10^{5} \mathrm{~Pa}$, the surface diameter of the nozzle $\mathrm{d}=40 \mathrm{~mm}$, the nozzle diameter $\mathrm{d}=10$ $\mathrm{mm}$, nozzle length $\mathrm{L}=15 \mathrm{~mm}$, tank diameter $\mathrm{D} 1=60 \mathrm{~mm}$, the depth $\mathrm{h} 1=2 \mathrm{~mm}$, the gap between nozzle and strip $\mathrm{h}=$ $0.3 \mathrm{~mm}$, the lower edge diameter of the nozzle $\mathrm{D}=120 \mathrm{~mm}$. The upper ring part of the nozzle has less impact on the characteristics of the nozzle. The ring part can be ignored to simplify the model. Three-dimensional geometric model and mesh are shown in Fig. (4).

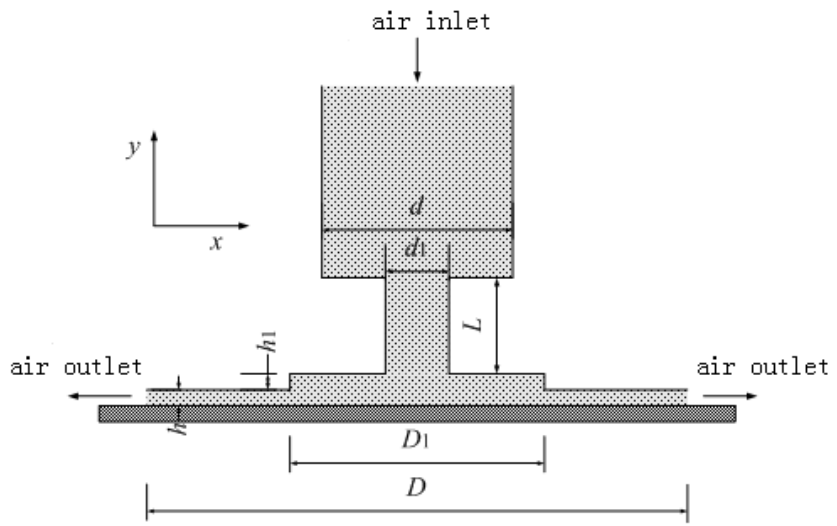

Fig. (3). Schematic diagram of the physical structure of the nozzle.

(a) An overall view of the model

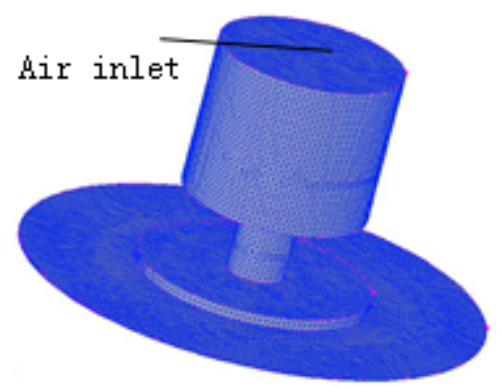

(b) A partial view of the model

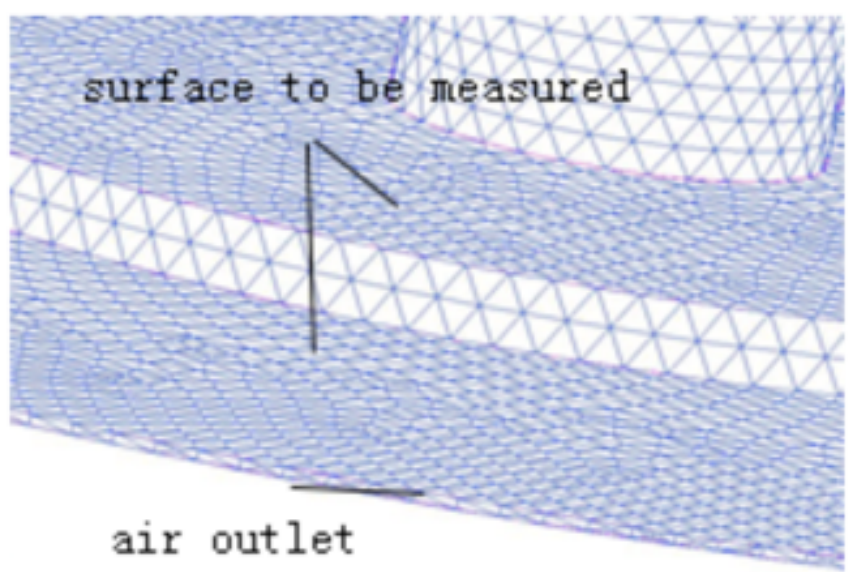

Fig. (4). The results of meshing the nozzle model.

\subsection{Boundary Conditions}

Boundary conditions for the flow field analysis are as follows. (1) inlet boundary: the air flow rate of the entrance is determined by the inlet pressure. $k$ can be taken as $0.4 \%$ of the flow energy, $\varepsilon$ is calculated according to the equation $\varepsilon=C_{D} \frac{k^{3 / 2}}{l}, l$ is taken as one tenth of the entrance diameter. (2) Outlet boundary: Air pressure at the outlet is equal to the pressure of the cooling zone. (3) solid wall boundary: the flow rate parallel to the wall is the axial velocity, $u_{w}=0$. 


\subsection{Simulation Results and Analysis}

\subsubsection{Impact of Equalizing Groove on the Nozzle Characteristics}

Fig. (5) shows the nozzle suspension system diagram without equalizing groove. Influence of equalizing groove on the reaction force of nozzle at different gap is researched. Fig. (6) shows the relationship between the reaction force and the gap. It can be seen from the figure that the reaction force on the nozzle reaches the maximum when the gap is about $0.2 \mathrm{~mm}$. If the gap is gradually reduced, the reaction force declines rapidly. When the gap is zero, the reaction force becomes zero. As the gap becomes larger, the reaction force is gradually decreased. Without the groove structure, the flow field pressure from the center to the outlet decays quite intensely. The average air pressure is less and it cannot purge the cooling water layer.

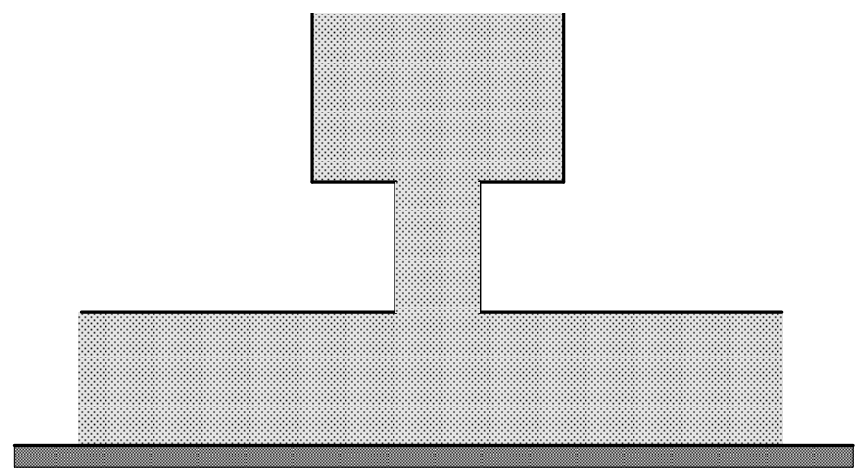

Fig. (5). The nozzle suspension system diagram (without equalizing groove).

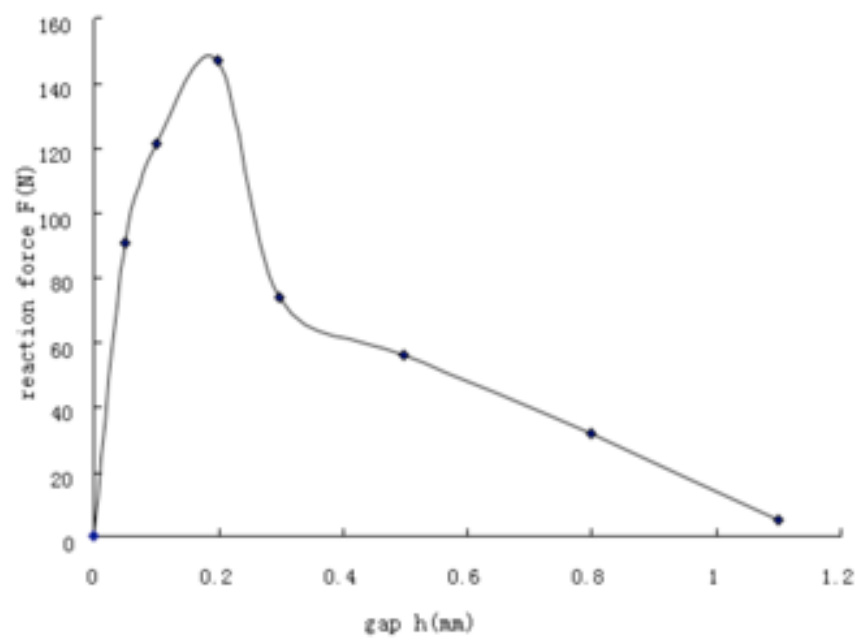

Fig. (6). The relationship between the reaction force and the gap (without equalizing groove).

Fig. (7) shows the nozzle suspension system diagram with equalizing groove. The relationship between reaction force and the nozzle gap is shown in Fig. (8). It can be seen from the Fig. (8) that as the gap increases, the reaction force is gradually decreased in the case of having a pressure groove. Therefore, pressure equalization groove can improve the performance of the nozzle in order to meet the design requirements.

Figs. $(\mathbf{9}, \mathbf{1 0})$ show the impact of the groove diameter and depth on the reaction force respectively. The nozzle cannot be suspended when the groove diameter D1 is less than $40 \mathrm{~mm}$. When $\mathrm{D}$ is greater than $60 \mathrm{~mm}$ having increased diameter, the reaction force changes a little. The nozzle cannot be suspended when the groove depth $\mathrm{h} 1$ is less than $0.9 \mathrm{~mm}$. When the groove depth $\mathrm{h} 1$ is bigger than $2 \mathrm{~mm}$, the reaction force changes a little.

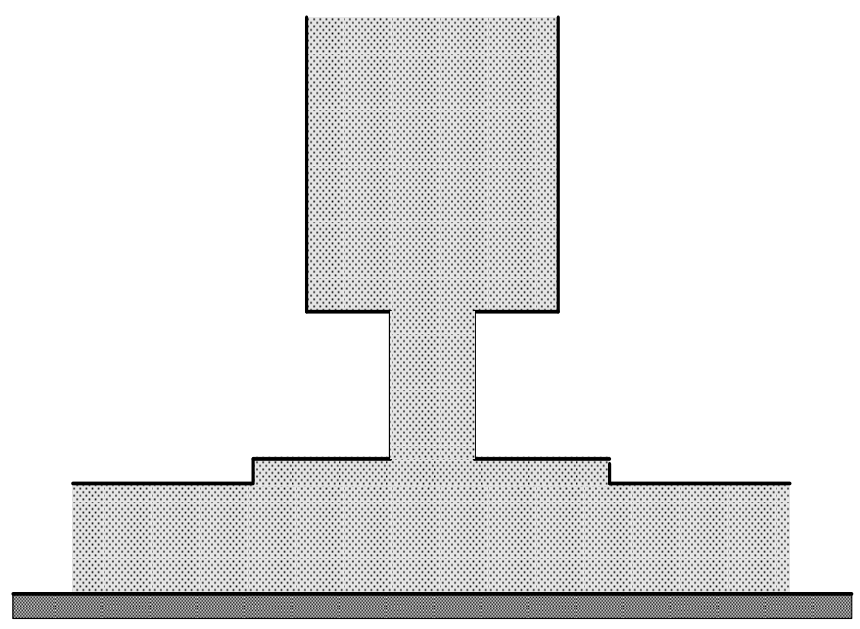

Fig. (7). The nozzle suspension system diagram (with equalizing groove).

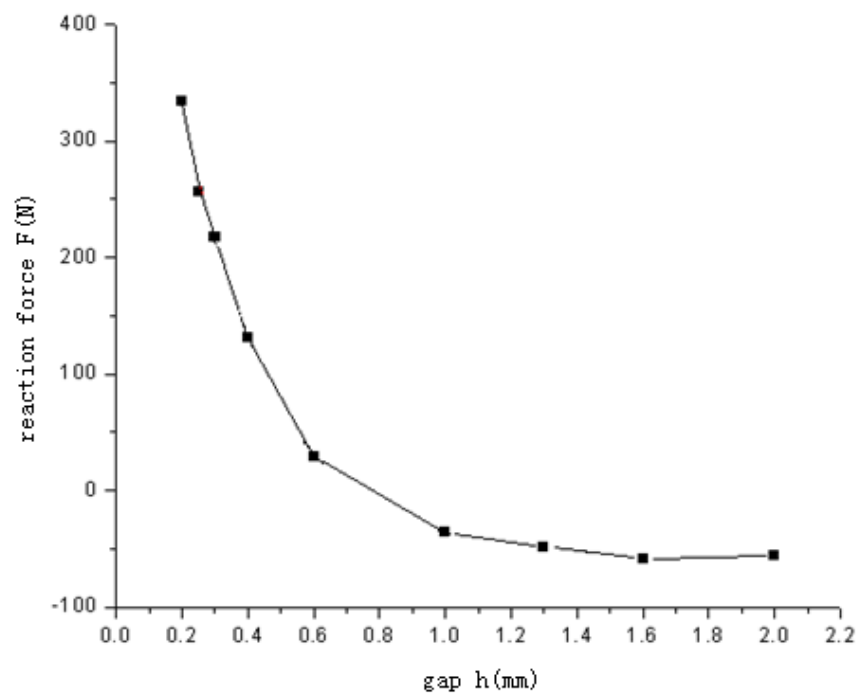

Fig. (8). The relationship between reaction force and the nozzle gap (with equalizing groove).

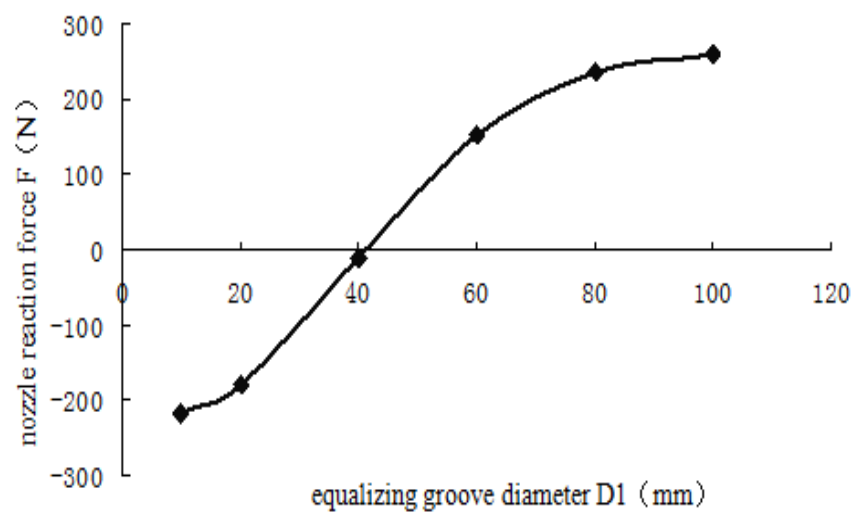

Fig. (9). The effect of equalizing groove diameter on the reaction force. 


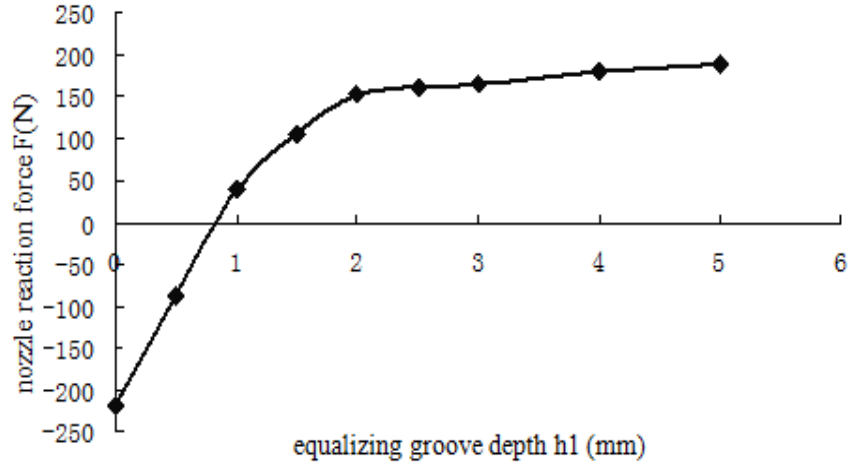

Fig. (10). The effect of equalizing groove depth on the reaction force.

\subsubsection{Impact of Nozzle Gap on the Flow Field Pressure Distribution}

Fig. (11) shows the flow field pressure contours inside the nozzle. Fig. (11a) shows the flow field pressure distribution inside the nozzle when the gap is equal to $0.2 \mathrm{~mm}$. Fig. (11b) shows the flow field pressure distribution inside the nozzle when the gap is equal to $0.5 \mathrm{~mm}$. Fig. (11c) shows the flow field pressure distribution inside the nozzle when the gap is equal to $0.8 \mathrm{~mm}$. The pressure on the nozzle surface gradually decreases with the increase in the gap $h$.

\subsubsection{Effect of Inlet Pressure}

When the gap $\mathrm{h}$ is equal to $0.3 \mathrm{~mm}$, the effect of inlet pressure on the reaction force of nozzle is shown in Fig. (12). It can be seen from Fig. (12) that the reaction force of the nozzle increases with the increase in inlet pressure. These are linear relationships. The force balance of nozzle cannot be changed by inlet pressure of nozzle. The effect of inlet pressure on the gas flow of the nozzle is shown in Fig. (13). It can also be seen from the figure that nozzle flow rate increases with the increase in inlet pressure.

\subsubsection{Influence of Nozzle Gap on Gas Flow}

The relationship between nozzle gas flow and the nozzle gap $h$ is shown in Fig. (14). It can be seen from the figure that the gas flow increases with the increase in the gap. As the gap exceeds a certain value, the flow changes steadily.

\subsubsection{Impact of the Lower Edge Diameter of the Nozzle}

The relationship between the lower edge diameter of the nozzle and the reaction force is shown in Fig. (15). It can be seen from the figure that the corresponding reaction force increases with the increase in lower edge diameter of the nozzle. The lower surface of the large nozzle easily collides with the steel strip surface mechanically. The machining accuracy and mounting accuracy become higher.

\subsubsection{The Impact of Nozzle Diameter}

Fig. (16) shows the pressure changes of steel strip surface with different nozzle diameters. The figure shows that the reaction force of large-diameter nozzle is significantly larger than that of the small diameter nozzle. In the case of large gaps, nozzle diameter can be increased to achieve a better sweep of the aqueous layer to meet the requirement of the temperature sensor. (a) $\mathrm{h}=0.2 \mathrm{~mm}$
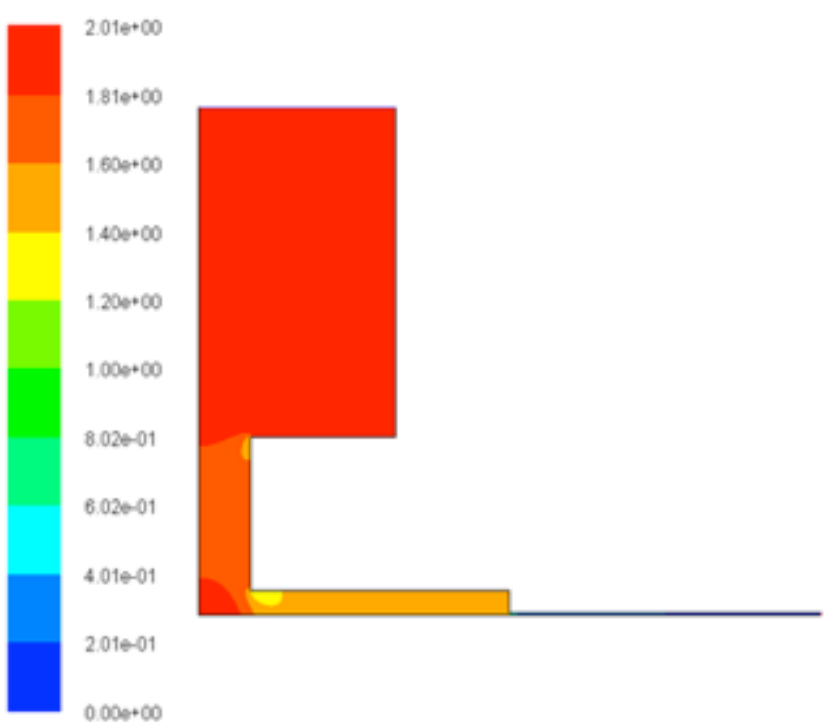

(b) $\mathrm{h}=0.5 \mathrm{~mm}$
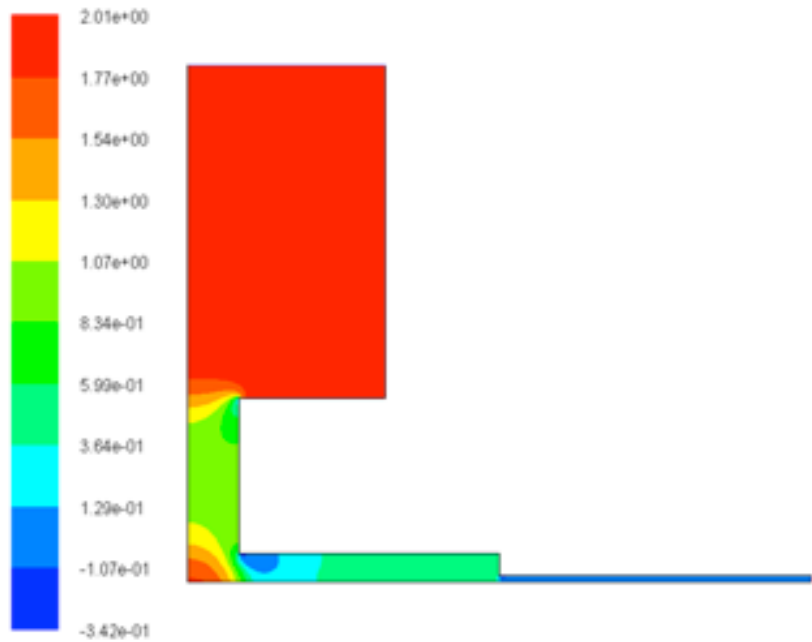

(c) $\mathrm{h}=0.8 \mathrm{~mm}$
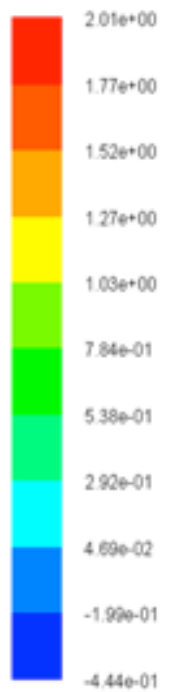

Fig. (11). The flow field pressure contours inside the nozzle. 


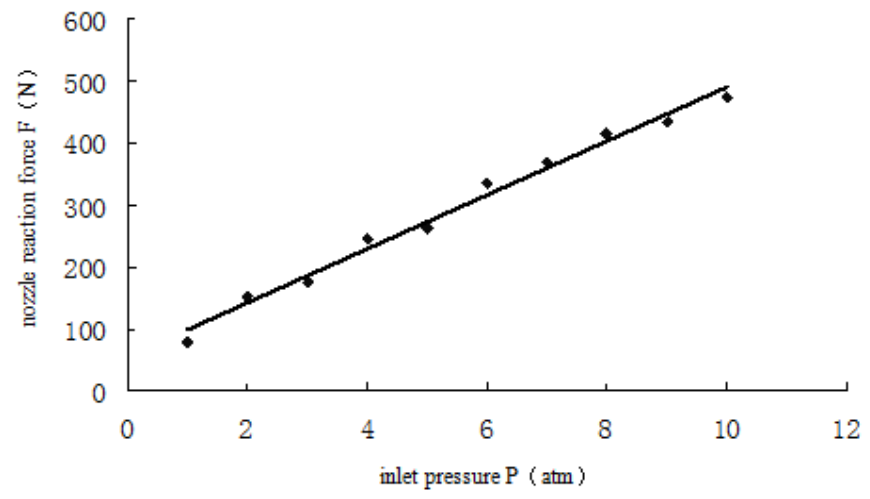

Fig. (12). The effect of inlet pressure on the reaction force of nozzle.

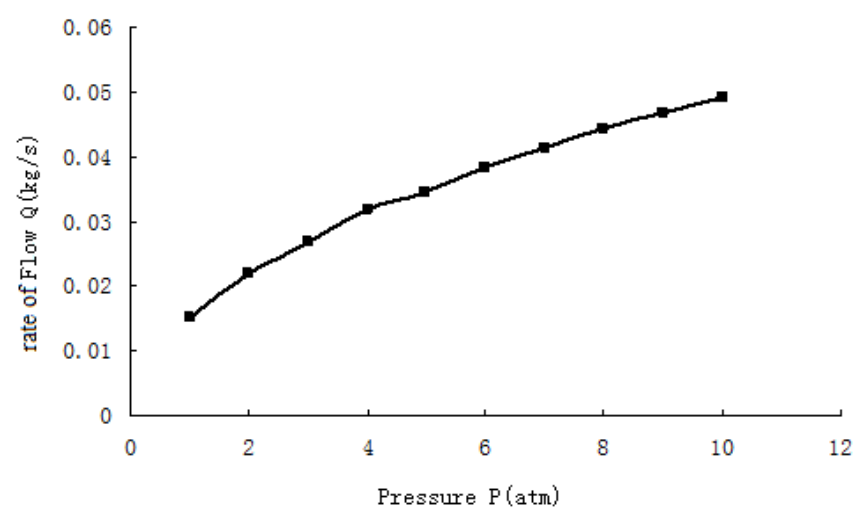

Fig. (13). The effect of inlet pressure on the gas flow of the nozzle.

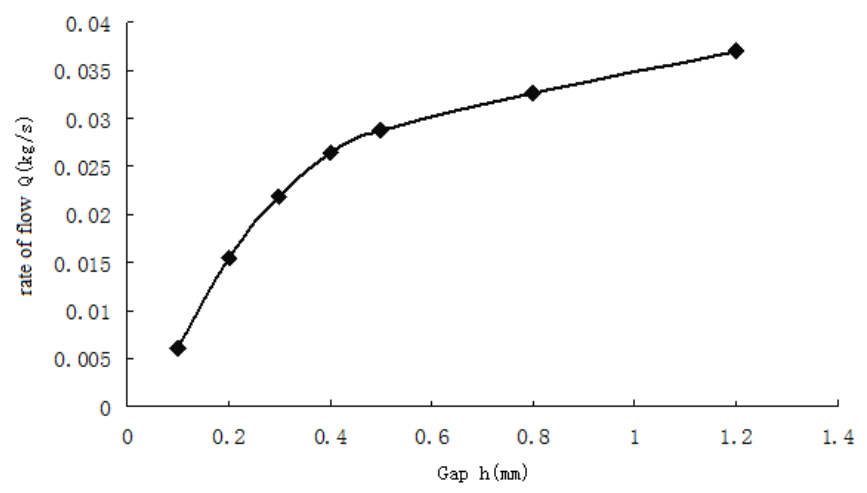

Fig. (14). The relationship between nozzle gas flow and the nozzle gap $h$.

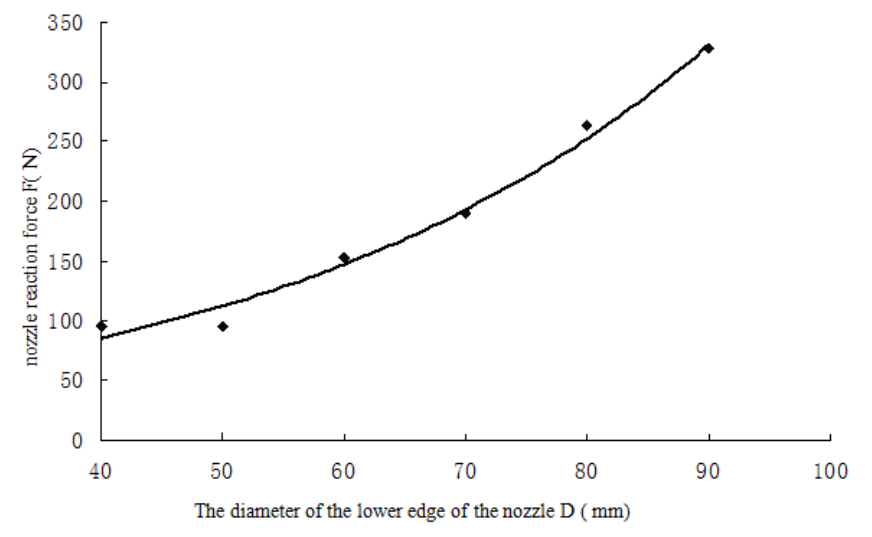

Fig. (15). The relationship between the lower edge diameter of the nozzle and the reaction force. (a) $\mathrm{h}=0.3 \mathrm{~mm}$

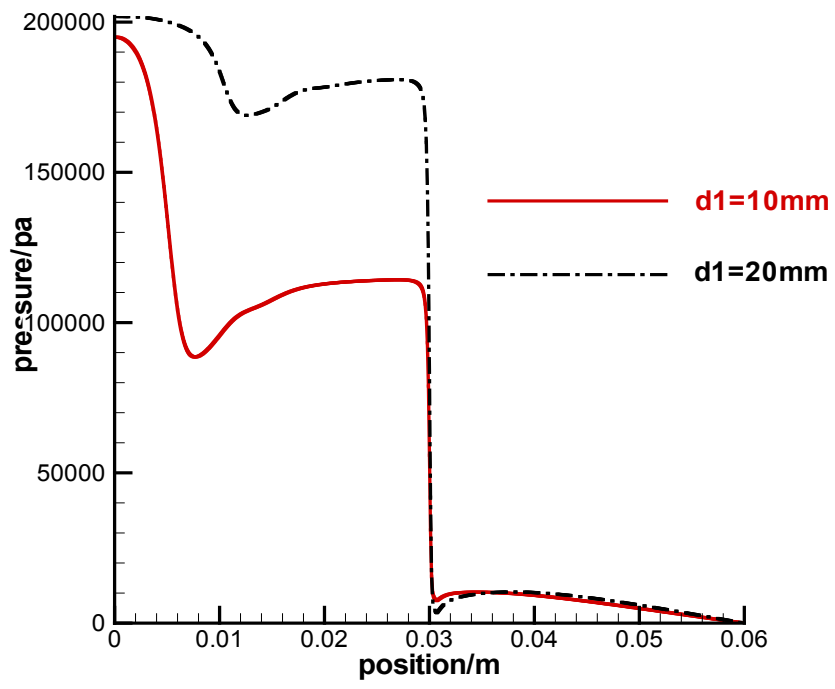

(a) $\mathrm{h}=0.5 \mathrm{~mm}$

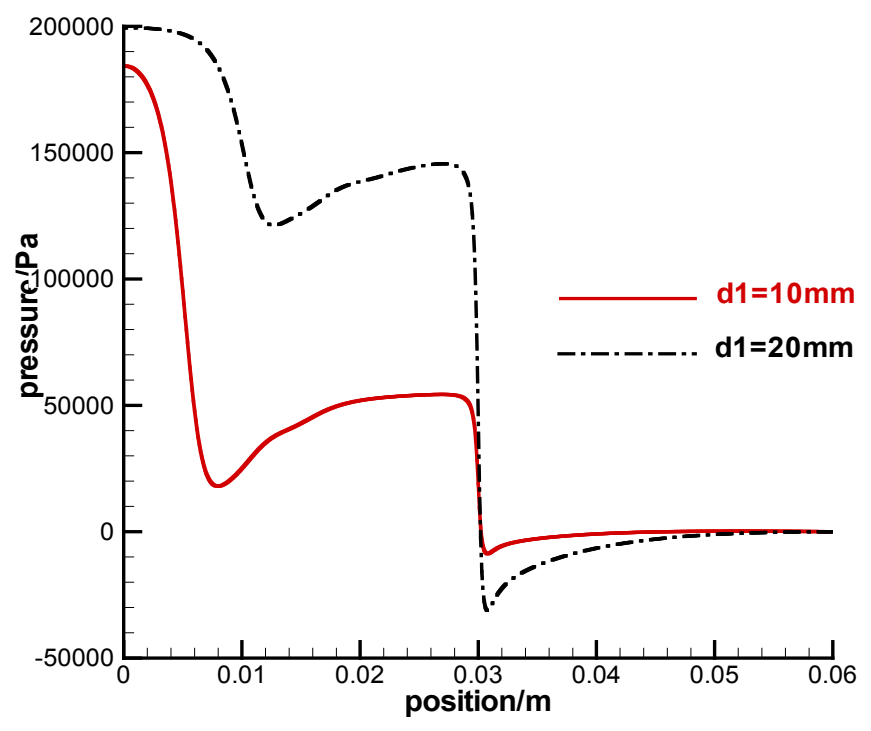

$\mathrm{h}=1 \mathrm{~mm}$

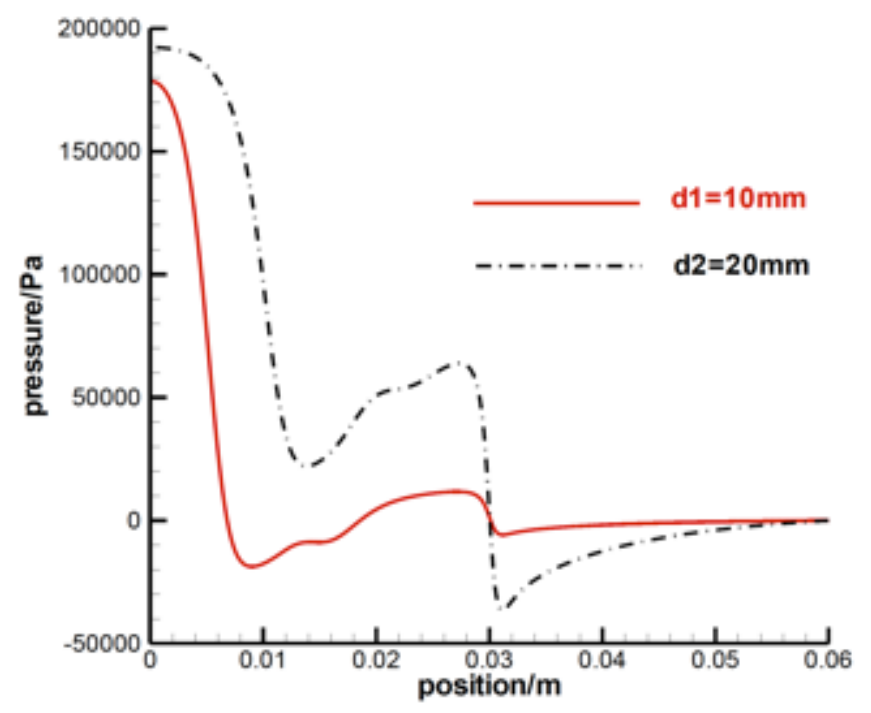

Fig. (16). The pressure changes of steel strip surface with different nozzle diameters. 


\subsubsection{Influence of Chamfer Structure}

Steady flow field is an important condition for ensuring suspension and purge of nozzle system. Fig. (17) shows the flow field pressure contour inside the nozzle without chamfer. Fig. (18) shows the flow field pressure contour inside the nozzle with chamfer. It can be seen from the pressure contours that the chamber pressure of purge is relatively stable. Chamfer structure can significantly improve and stabilize the pressure inside the nozzle. Great pressure fluctuations occur near the nozzle border without chamfer structure and vortex phenomenon is generated. Chamfer structure can reduce pressure fluctuations and can effectively prevent the occurrence of the vortex.
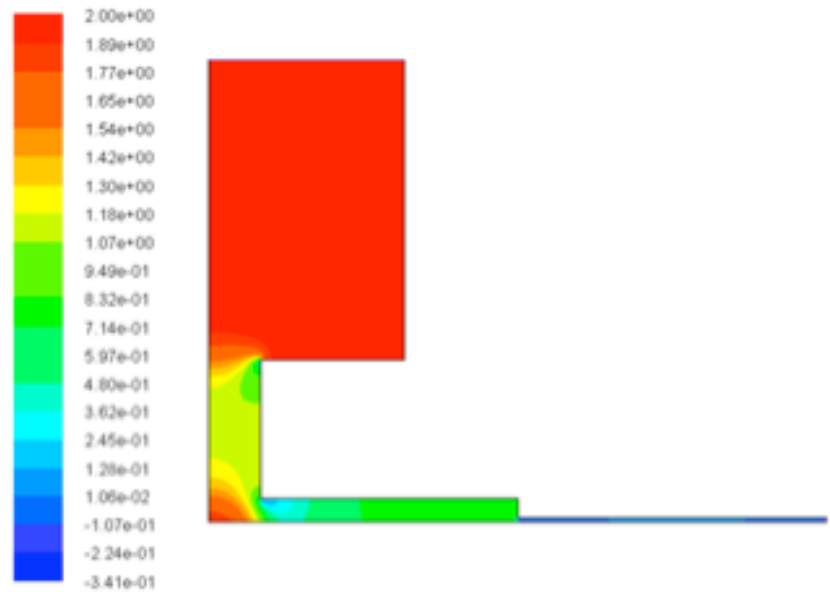

Fig. (17). The flow field pressure contour inside the nozzle without chamfer.

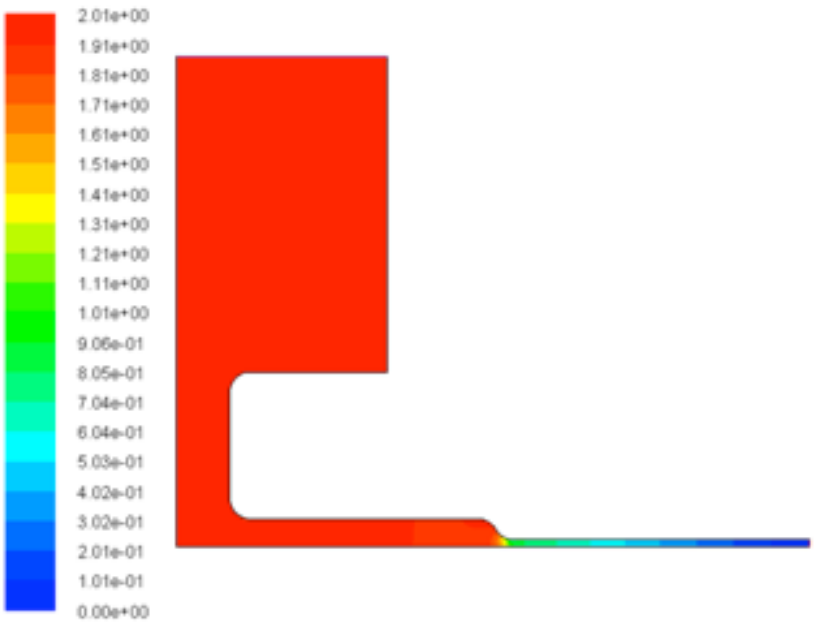

Fig. (18). The flow field pressure contour inside the nozzle with chamfer.

\section{CONCLUSION}

1) A new hot steel strip temperature measurement device is designed. The temperature measuring device is a portable non-contact temperature measuring device.
2) The nozzle without equalizing groove cannot achieve the function of purging the cooling water. Equalizing groove can improve the performance of the nozzle so as to meet the design requirements.

3) Within the scope of the work space, the reaction force of steel strip increases with the decrease in nozzle gap. Nozzle flow rate increases when the inlet pressure of nozzle increases. Gas flow increases along with the increase in nozzle gap. With the increase in the lower edge diameter of the nozzle, the reaction force correspondingly increases. Increasing the diameter of the nozzle can better achieve the effect of purging the water layer. The chamfer structure of the nozzle can reduce pressure fluctuation and effectively prevent the occurrence of the vortex so as to get a more stable flow field.

\section{CONFLICT OF INTEREST}

The authors confirm that this article content has no conflict of interest.

\section{ACKNOWLEDGEMENTS}

This paper is supported by the National Natural Science Foundation of China (Grant No.51205336), the Natural Science Foundation of Fujian Province (Grant No. 2013J05086) and the Science and Technology Plan Project of Fujian Province (Grant No. 2013H0050).

\section{REFERENCES}

[1] B. H. Tan, Z. H. Feng, D. D. Liu, and Y. P. Tang, "Flow field analysis of auxiliary nozzle of air-jet loom based on CFD," J. Textile Res., vol. 33, pp. 123-128, 2012.

[2] H. Ruan, J. Lv, B. Yang, X. Wang, and H. Liu, "Analysis of flow field for supersonic nozzle in laser cutting," Chinese J. Lasers, vol. 5, pp.1233-1238, 2009.

[3] J. Hu, S. Guo, M. Qiu, and Z. Yao, "Analysis of assistant gas flow field and structure improvement of supersonic nozzle in laser cutting," $J$. Mech. Eng., vol. 45, pp. 251-255, 2009.

[4] L. Xinping, X. Yao, and X. L. Yang, "Flow simulation and vortex structure analysis of multi-nozzle jet pumps," J. Drainage Irrigat. Mach. Eng., vol. 30, pp. 136-140, 2012.

[5] S. Xing, C. C. Ma, and W. Lu, "The investigation on characteristics of nozzle incidence of JK90S variable nozzle turbine," J. Harbin Eng. Uni., vol. 34, pp. 635-640, 2013.

[6] J. Liu, S. Xu, and Y. Lou. "Simulation of flow field of abrasive waterjet from nozzle with CFX," Oil Field Equip., vol. 28, pp. 40-44, 2013.

[7] G. Wang, K. Yang, and H. Liang, "Analysis of flow field of the main nozzle of air-jet loom," J. Xi'an Polytech. Univ., vol. 22, pp. 424-426, 2008

[8] W. Xu, J. Ma, and S. Deng, "Analysis and research on waterjet field of high pressure spray gun clean tank robot based on FLUENT," Robot Tech. Appl., vol. 25, pp. 30-33, 2012.

[9] G. Jian, H. Shen, M. Sun, and Z. Liang. "CFD modeling of an innovative ejector." Fluid Mach., vol.37, pp. 25-28, 2009.

[10] Q. Chen, and G. Zhang, "Numerical simulation on flow field in spray nozzle of coal shearer." Coal Sci. Tech., vol. 34, pp. 25-26, 2006.

[11] Z. Zhang, and Q. F. Zhang "Study on jet simulation of high pressure nozzle." Mech. Elect. Eng. Mag., vol. 30, pp. 185-188, 2013.

[12] M. Tian, and S. Jing. "The structural improvements and analysis for flow field of ejector booster pump."Chem. Equip. Tech., vol. 26, pp. 5861, 2005.

This is an open access article licensed under the terms of the Creative Commons Attribution Non-Commercial License (http://creativecommons.org/licenses/ by-nc/4.0/) which permits unrestricted, non-commercial use, distribution and reproduction in any medium, provided the work is properly cited. 